\title{
Matched filter in low-number count Poisson noise regime: Efficient and effective implementation
}

\author{
R. Vio ${ }^{1}$ and P. Andreani ${ }^{2}$ \\ ${ }^{1}$ Chip Computers Consulting s.r.l., Viale Don L. Sturzo 82, S. Liberale di Marcon, 30020 Venice, Italy \\ e-mail: robertovio@tin.it \\ 2 ESO, Karl Schwarzschild strasse 2, 85748 Garching, Germany \\ e-mail: pandrean@eso.org
}

Received 14 January 2018 / Accepted 15 April 2018

\begin{abstract}
The matched filter (MF) is widely used to detect signals hidden within the noise. If the noise is Gaussian, its performances are wellknown and can be described in an elegant analytical form. The treatment of non-Gaussian noises is often cumbersome as in most cases there is no analytical framework. This is true also for Poisson noise which, especially in the low-number count regime, presents the additional difficulty to be discrete. For this reason in the past methods have been proposed based on heuristic or semi-heuristic arguments. Recently, an analytical form of the MF has been introduced but the computation of the probability of false detection or false alarm (PFA) is based on numerical simulations. To overcome this inefficient and time-consuming approach, we propose here an effective method to compute the PFA based on the saddle point approximation (SA). We provide the theoretical framework and support our findings by means of numerical simulations. We also discuss the limitations of the MF in practical applications.
\end{abstract}

Key words. methods: data analysis - methods: statistical - methods: numerical

\section{Introduction}

Detecting signals embedded in noise is a challenge in many research and engineering areas. In the common case of Gaussian noise the standard tool is the matched filter (MF; Tuzlukov 2001; Minkof 2002; Osche 2002; Briggs 2004; Levy 2008; Yao et al. 2013). According to the Neyman-Pearson theorem, the MF provides the greatest probability of true detection under the condition of a constant probability of false detection (e.g., Kay 1998). In non-Gaussian cases it is difficult to derive an MF, and even if available it is more cumbersome to use. In particular, it is complex to compute the probability of false detection or false alarm (PFA). In astronomy this situation occurs, for example, in the search for high-energy point sources in the presence of Poisson (i.e., non-additive) noise and where signal and noise together consist of only a few counts per pixel. For this reason, in the past alternative procedures based on heuristic or semi-heuristic arguments have been preferred (Stewart 2006, and reference therein). In a recent work, Ofek \& Zackay (2017) provide an analytical form of the MF with the PFA computed from numerical simulations. This approach lacks flexibility and is time-consuming. In the present paper, we propose a method to compute the PFA based on the saddle-point approximation (SA) which is fast, flexible, and provides accurate results.

In Sect. 2, the problem of the detection of a signal in Poisson noise is formalized whereas the SA method is described in Sect. 3 and its application illustrated in Sect. 4. The results of a few numerical experiments are given in Sects. 5 and 6 and the limitations of the MF in practical situations are discussed in Sect. 7. Finally, the conclusions are given in Sect. 8.

\section{Mathematics of the problem}

In this section, we describe the basic properties of the MF in the case of Poisson noise. To allow a better understanding, we have developed the main arguments in one dimension. The extension to higher dimension cases is straightforward and can be done by substituting the coordinate system with a multidimensional one ${ }^{1}$.

To illustrate the procedure of detection of a deterministic and discrete signal of known structure $s=[s(0), s(1), \ldots, s(N-1)]^{T}$, with length $N$, and the symbol ${ }^{T}$ denoting a vector or matrix transpose, we assume the following:

- the searched signal takes the form $\boldsymbol{s}=a \boldsymbol{g}$ with $a$ a positive scalar quantity (amplitude) and $\boldsymbol{g}$ typically a smooth function often somehow normalized (e.g., $\sum_{i=0}^{N-1} g(i)=1$ );

- the signal $s$ is contaminated by a Poisson noise, meaning that the observed signal $\boldsymbol{x}$ is given by $\boldsymbol{x}=\mathcal{P}(\boldsymbol{s}+\lambda \mathbf{1})$. Here, $\mathbf{1}=[1,1, \ldots, 1]^{T}$, the scalar $\lambda$ represents the intensity parameter of the noise background and $\mathcal{P}(\mu \mathbf{1})$ denotes a Poisson random vector with independent entries and expected value (i.e., mean) $\mathrm{E}[\boldsymbol{x}]=\mu$. Although not necessary in what follows, this implies that $\lambda$ is constant across $\boldsymbol{x}$.

Under these conditions, the detection problem consists of deciding whether $\boldsymbol{x}=\boldsymbol{n}=\mathcal{P}(\lambda \mathbf{1})$, i.e., it is pure noise (hypothesis $H_{0}$ ), or it does contain a contribution from a signal $s$ (hypothesis $H_{1}$ ). In this way, it is equivalent to a decision problem between

\footnotetext{
1 In the case of a two-dimensional map $X$, a one-dimensional signal $\boldsymbol{x}$ is obtained through $\boldsymbol{x}=\operatorname{VEC}[\boldsymbol{X}]$, with VEC[.] the operator that transforms a matrix into a column array by stacking its columns one underneath the other.
} 
the two hypotheses

$$
\begin{cases}\mathcal{H}_{0}: & \boldsymbol{x}=\mathcal{P}(\lambda \mathbf{1}) \\ \mathcal{H}_{1}: & \boldsymbol{x}=\mathcal{P}(\boldsymbol{s}+\lambda \mathbf{1})\end{cases}
$$

Under $\mathcal{H}_{0}$ the probability density function (PDF) of $\boldsymbol{x}$ is given by $p\left(\boldsymbol{x} \mid \mathcal{H}_{0}\right)$ whereas under $\mathcal{H}_{1}$ by $p\left(\boldsymbol{x} \mid \mathcal{H}_{1}\right)$. Deciding between these two alternatives requires fixing the detection criterion. A common and effective criterion consists in maximizing the probability of detection (PD) under the constraint that the PFA (i.e., the probability of a false detection) does not exceed a fixed value $\alpha$. The Neyman-Pearson theorem (e.g., see Kay 1998) allows us to design a decision process that pursues this aim: to maximize the PD for a given PFA $=\alpha$, choose $\mathcal{H}_{1}$ if the likelihood ratio

$L(\boldsymbol{x})=\frac{p\left(\boldsymbol{x} \mid \mathcal{H}_{1}\right)}{p\left(\boldsymbol{x} \mid \mathcal{H}_{0}\right)}>\gamma$,

where the threshold $\gamma$ is found from

$\mathrm{PFA}=\int_{\{x: \mathrm{L}(\boldsymbol{x})>\gamma\}} p\left(\boldsymbol{x} \mid \mathcal{H}_{0}\right) \mathrm{d} \boldsymbol{x}=\alpha$.

In a recent work, Ofek \& Zackay (2017) show that criteria (2) and (3) lead to the test

$T(\boldsymbol{x})=\boldsymbol{x}^{T} \mathfrak{\mathfrak { f }}>\gamma$,

where

$\mathfrak{f}=\ln \left(\mathbf{1}+\frac{\boldsymbol{s}}{\lambda}\right)$.

In practice, the test consists of filtering the signal $\boldsymbol{x}$ with $\mathfrak{f}$ (i.e., the matched filter) and checking if the statistic $T(x)$ exceeds the threshold $\gamma$. From Eq. (5) it appears that the form of $\mathfrak{f}$ depends on the signal $s$. The issue here is that in many practical applications (e.g., detection of point sources in sky maps) only the template $\boldsymbol{g}$ of the signal is known but not its amplitude $a$. The consequence of using the MF with an incorrect amplitude $a$ is to reduce, for a fixed value of the PFA, the PD, i.e., to make the MF less efficient. Ofek \& Zackay (2017) have shown that the results provided by the MF are not very sensitive to the precise value of $a$ (see also below). This is not surprising given the logarithmic dependence of $\mathfrak{f}$ on $\lambda$. However, even for a specific value of $a$, the PDF of $T(\boldsymbol{x})$ under the hypothesis $\mathcal{H}_{0}$ is not available in analytical form. The reason is that, although $T(\boldsymbol{x})$ is given by a linear composition of Poisson random variables, its PDF is not Poissonian. This does not allow fixing the threshold $\gamma$ corresponding to a prefixed value $\alpha$ of the PFA. Ofek \& Zackay (2017) bypass this problem using numerical simulations. Such a method, however, is not flexible and is time-consuming.

An alternative to numerical simulations is approximating the unknown PDF of $T(\boldsymbol{x})$ by another PDF. For example, in the situations of high-number count noise regimes, the Gaussian PDF would be a good choice. The same does not hold in lownumber count regimes. In this case the SA represents an effective solution.

\section{Saddlepoint approximation basics}

The SA is a powerful tool, able to provide accurate expressions of the PDFs and the corresponding cumulative distribution functions (CDF). Its derivation is rather technical. For this reason, we provide here only a basic introduction useful for practical applications. A simple and informal derivation is outlined in Appendix A, whereas a rigorous derivation is given in Butler (2007).

The SA to the PDF $f_{X}(x)$ of a continuous random variable $X$ is given by

$\hat{f}_{X}(x)=\frac{1}{\sqrt{2 \pi K_{X}^{(2)}(\hat{s})}} \exp \left(K_{X}(\hat{s})-\hat{s} x\right)$,

where $K_{X}(s)$ is the cumulant generating function (CGF) of $f_{X}(x)$,

$K_{X}(s)=\ln M_{X}(s)$,

and $M_{X}(s)$ the corresponding moment generating function (MGF; see Appendix B). Moreover, $\hat{s}$ is the unique solution to the equation

$K_{X}^{(1)}(\hat{s})=x$,

with $K_{X}^{(j)}$ denoting the $j$ th derivative with respect to $s . \hat{f}_{X}(x)$ will not, in general, integrate to one, although it will usually not be far off. Therefore, it has to be numerically normalized.

The SA results are particularly useful to approximate PDFs that cannot be obtained in analytical form for which the corresponding CGF is available. This situation is typical of a random variable $X$ given by the sum of a set of independent random variables $\left\{X_{i}\right\}, i=1, \ldots, N$. Indeed, except for special cases (e.g., Gaussian), even when the random variables $\left\{X_{i}\right\}$ share the same PDF, it is not possible to obtain $f_{X}(x)$ in closed form. As explained in Appendix B, the CGF of a sum of independent random variables is given by the sum of the respective CGFs. Hence, if the CGFs of the random variables $\left\{X_{i}\right\}$ are available, the SA can be applied.

Once that $\hat{f}_{X}(x)$ is available, the correspoding $\operatorname{CDF} \hat{F}_{X}(x)$ can be obtained via numerical integration. However, a simple expression, able to provide excellent results, has been proposed by Lugannani \& Rice (1980)

$\hat{F}_{X}(x)= \begin{cases}\Phi(\hat{w})+\phi(\hat{w})(1 / \hat{w}-1 / \hat{u}), & \text { if } x \neq \mu, \\ \frac{1}{2}+\frac{K_{X}^{(3)}(0)}{6 \sqrt{2 \pi} K_{X}^{(2)}(0)^{3 / 2}}, & \text { if } x=\mu .\end{cases}$

Here, $\phi($.$) and \Phi($.$) represent the standard Gaussian PDF and$ $\mathrm{CDF}$, respectively, whereas $\hat{w}$ and $\hat{u}$ are given by

$\hat{w}=\operatorname{sign}(\hat{s}) \sqrt{2\left[\hat{s} x-K_{X}(\hat{s})\right]}$,

with $\operatorname{sign}(y)$ providing the sign of $y$, and

$\hat{u}=\hat{s} \sqrt{K_{X}^{(2)}(\hat{s})}$.

The only difficulty in using $\hat{F}_{X}(x)$ concerns its evaluation when $x$ is close to the expected value $E[X]=\mu$ of $X$. In this case the computation of $\hat{F}_{X}(x)$ is tricky since $K_{X}^{(1)}(0)=E[X]$ and then the solution of Eq. (8) becomes $\hat{s}=0$. As a consequence, it happens that $K_{X}(0)=0$ and accordingly, since $\hat{w}=0$ from Eq. (10), the first equation in the system (9) becomes useless. This is the reason for the second equation in the system (9). For practical use, however, it is numerically more advantageous to use a linear interpolation based on the SA to $E[X] \pm \epsilon$, where $\epsilon$ is chosen 
small enough to ensure high accuracy, but large enough to ensure numerical stability.

In the case for which $X$ is a discrete random variable, the same equations hold with $x$ substituted by $k$, which takes values in the set of the integer numbers, and keeping in mind that $1-\hat{F}_{X}(k)$ provides the probability that $X \geq k$. Although the expressions Eqs. (6) and (9) are computable for any value of $x$ whether real or integer-valued, $\hat{f}_{X}(k)$ and $\hat{F}_{X}(k)$ are meaningful approximations of $f_{X}(k)$ and $F_{X}(k)$ only for integer-valued arguments.

\section{Signal detection in the Poisson noise regime}

The SA to the PDF and the CDF of $T(x)$ in the Poisson case can be easily computed. Indeed, under the hypothesis $\mathcal{H}_{0}, T(\boldsymbol{x})$ is given by a linear combination (i.e., a sum) of Poisson random variables $X_{i}$ with common parameter $\lambda$,

$T(\boldsymbol{x})=y=\sum_{i=0}^{N-1} \mathfrak{f}_{i} x_{i}$.

Hence, its $\mathrm{CGF} K_{Y}(s)$ is given by

$$
\begin{aligned}
K_{Y}(s) & =\sum_{i=0}^{N-1} K_{X_{i}}(s) \\
& =\lambda \sum_{i=0}^{N-1}\left(\mathrm{e}^{\mathfrak{f}_{i} s}-1\right),
\end{aligned}
$$

with $s \in(-\infty,+\infty)$. It is elementary to see that $K_{Y}^{(j)}(s)$ is given by

$$
K_{Y}^{(j)}(s)=\lambda \sum_{i=0}^{N-1} \mathfrak{f}_{i}^{j} \mathrm{e}^{\mathfrak{f}_{i} s} .
$$

To be used in Eqs. (6) and (9), these functions have to be computed for $s=\hat{s}$. This step requires the numerical solution of Eq. (8) that, however, does not present particular difficulties given that, as explained in Appendix A, $K_{X}^{(1)}(s)$ is an increasing function for $s \in(-\infty,+\infty)$.

It is important to stress that, since Eq. (8) cannot be solved when $x=0, \hat{f}_{X}(x)$ is defined only for $x>0$. But it is easy to see that for $x=0$ it is $\hat{f}_{X}(0)=\exp (-\lambda N)$. This last one is an exact result not only an approximation.

When using the SA in the present context, it is necessary to consider that the PDF of $T(\boldsymbol{x})$ is of discrete type but it is not defined on a lattice (i.e., $y$ does not take values on a regular grid of numbers). The point is that the arguments presented above hold only for integer values of $k$ or, with minor modifications, when $k$ takes values on a regular grid of numbers. However, except for extremely small values of $\lambda$, this PDF can be considered "almost continuous". Indeed, in the case of two-dimensional maps, from combinatorial arguments it can be realized that, already with values of $\lambda$ as small as 0.01 and $\mathfrak{f}$ given by a circular Gaussian with standard deviation $\sigma$ of only two pixels, the number of different values that $y$ can take is on the order of several thousands. In any case, there is considerable empirical evidence that the SA is useful and maintains most of its accuracy even with discrete PDFs that are not defined on a lattice (cf. p. 27 in Butler 2007). The numerical simulations presented below confirm this result.

\section{Numerical experiments}

Figure 1 compares the histogram $H(y)$ with the SA $\hat{f}_{Y}(y)$ (normalized to unit area) to the PDF $f_{Y}(y)$ of the statistic $T(\boldsymbol{x})$ for the central pixel of a set of $10^{5} \mathrm{MF}$ filtered random realizations of a Poisson $13 \times 13$ pixels noise process. Four values of the parameter $\lambda$, say $0.01,0.025,0.05$, and 0.1 (units in counts pix ${ }^{-1}$ ), have been considered. The MF has been computed assuming $\boldsymbol{s}$ as a circular Gaussian with standard deviation $\sigma=2$ pixels normalized to unit volume. For comparison, the Gaussian best fits $\phi(y)$ are also shown. From these figures it is visible that better results are obtainable when increasing the values of $\lambda$. This is not an unexpected result since small values of $\lambda$ imply that most of the pixels have zero counts, a few have one count and very few have larger counts. Especially for "narrow" signals $s$, the consequence is a rough PDF for the statistic $T(\boldsymbol{x})$. However, in the case of $\lambda=0.01$, the top-right panel of Fig. 1 shows that, although the SA is not able to reproduce all the details of $H(y)$, it provides a good envelope resulting in a good approximation to the corresponding CDF. This is supported by Fig. 2 which compares the sample CDF $\tilde{F}_{Y}(y)$ with the $\hat{F}_{Y}(y)$ corresponding to the PDFs in Fig. 1. The agreement is excellent. This is more evident in Fig. 3 where the relative errors $\left(\hat{F}_{Y}(y)-\tilde{F}_{Y}(y)\right) / \tilde{F}_{Y}(y)$ are plotted versus $\tilde{F}_{Y}(y)$ for values of $y$ such as $\tilde{F}_{Y}(y)>0.7$. This is a useful fact since in signal detection problems it is the complementary CDF $1-F_{Y}(y)$ and not the PDF $f_{Y}(y)$ which matters.

The SA does not work with very small values of $\lambda$. However, it is questionable that in situations of very low-number count regime the MF could be a useful approach. For example, in the case of a $1000 \times 1000$ pixels map and $\lambda=0.001$, only $N_{\emptyset}=1000$ pixels are expected with values greater than zero, in other words, only one pixel in each area of $30 \times 30$ squared pixels. Under these conditions the use of the MF does not make sense since there is nothing to filter out. Moreover, the expected noise background should consist of 1000 bumps all with the same shape and amplitude. A more effective approach is based on the observation that the probability to have two counts in a pixel is on the order of $5 \times 10^{-7}$. Because of this, all the non-zero pixels are expected to have only one count. In addition, given that the probability of two pixels occupying adjacent positions is of order of $4 N_{\emptyset} / N_{\text {pix }}$, only four of them are expected to be neighboring. In other words, the detection of a signal can be claimed with high confidence in presence of pixels with counts greater than one and possibly contiguous with other non-zero pixels.

The conclusion is that, apart from extremely low levels of the noise background, the SA is able to provide excellent results. As visible in Figs. 1-3, this is not true for the Gaussian approximation. Hence, to test if after the MF filtering the value $y$ of a pixels is not due only to the noise, it is sufficient to check if $1-\hat{F}_{Y}(y)<\alpha$ with $\alpha$ a prefixed PFA.

\section{Expected performances of the MF}

The excellent computation efficiency, but above all the good flexibility of the SA in the calculation of the PFA with different noise levels and functional forms of $s$, allow us to explore the expected performances of the MF in better detail than is possible with an approach based on numerical simulations. In particular, in Ofek \& Zackay (2017), the performances of the MF are compared to those of its most important competitor which is the point spread function filter (PSFF) technique where it is assumed that $\mathfrak{f}=\boldsymbol{g}$. One of the main reasons for such a comparison lies in the fact that in the case of a Gaussian additive white-noise the PSFF and the MF concide. Another reason is that even for 

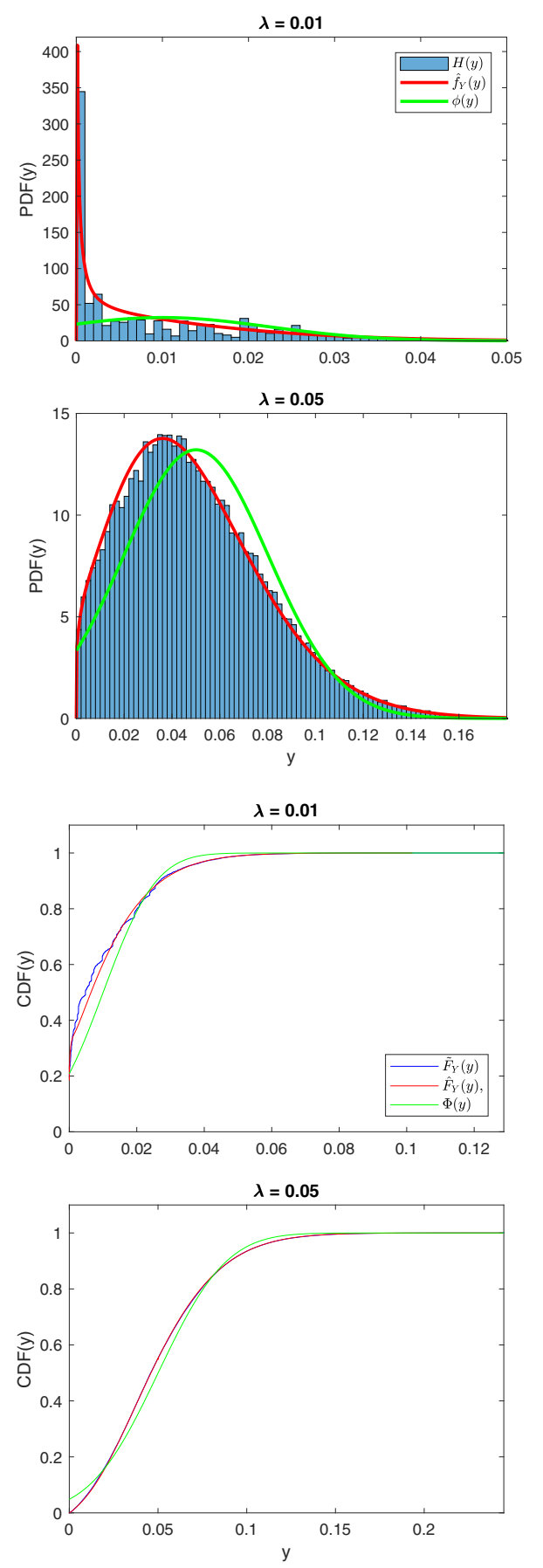
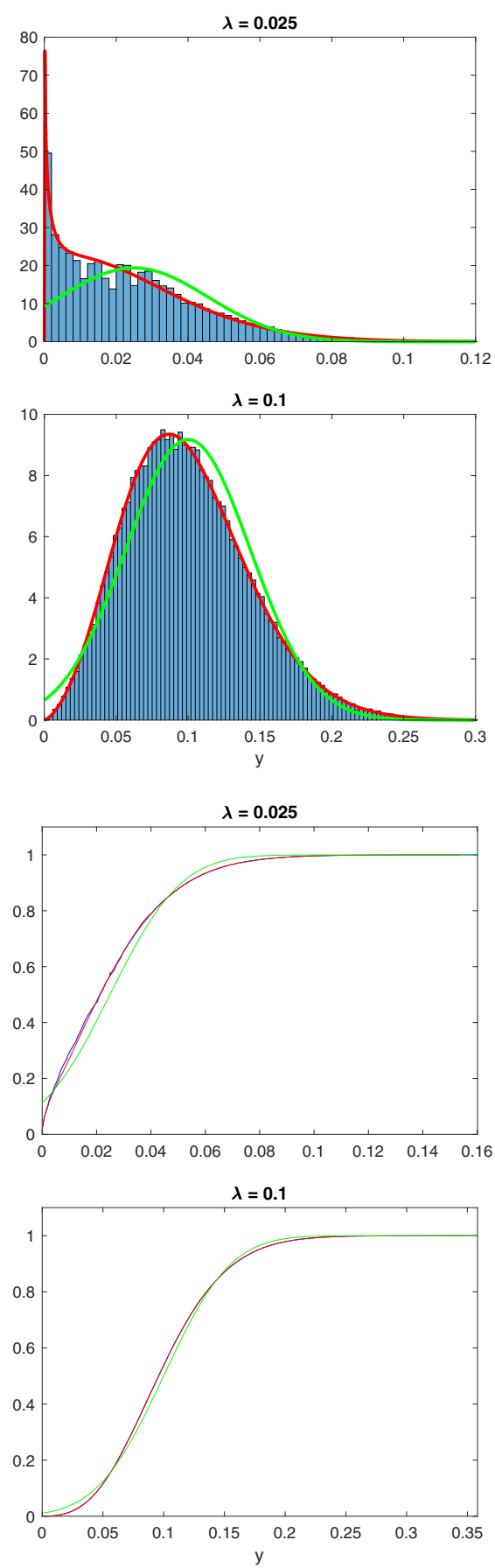

Fig. 1. PDF in the case of the SA (red curve) and the best Gaussian fit (green curve) of the PDF of the statistic $y=T(\boldsymbol{x})$ vs. the corresponding histogram $H(y)$ obtained with a set of $10^{5} \mathrm{MF}$ filtered random realizations of a Poisson $13 \times 13$ pixels noise process with the parameter $\lambda$ set to $0.01,0.025,0.05$, and 0.1 counts pix $^{-1}$ (see Sect. 5 for details). The used MF is given by Eq. (5) with $s$ a circular Gaussian with standard deviation $\sigma=2$ pixels normalized to unit volume. relatively small values of $\lambda$ the PDF of the Poisson noise can be reasonably approximated with a Gaussian. Moreover, under the conditions we are working under, the Poissonian noise level is constant across the background area but it changes only close to the signal position. However, since the MF is often used in situations where the amplitude of the signal is smaller than the level of the noise, in first approximation it can be assumed that $\boldsymbol{s}+\lambda \mathbf{1} \approx \lambda \mathbf{1}$. Hence, the noise can be considered of additive type with a constant level everywhere. In conclusion, there are situations where the PSFF $\boldsymbol{g}$ can be an acceptable approximation to the MF. The results obtained by Ofek \& Zackay (2017) indicate that in general the MF is superior to the PSFF for small values of $\lambda$ and similar for larger values of this parameter. But, because of the very large number of numerical simulations necessary to fix a reliable detection threshold for each different experimental situation, their analysis is based on a few cases only. Here, we carry out a set of similar simulations with a larger combination of noise levels and signal intensities.

Our numerical experiments confirm the results of Ofek \& Zackay (2017). This is shown in Fig. 4 where the completeness (i.e., the fraction of correctly detected signals) of the MF is compared with that of the PSFF for various combinations of $\lambda$ and $a$. It is visible as the results provided by the MF are effectively superior to those of the PSFF. However, the performances move closer and closer for increasing values of $\lambda$. In the experiments $\boldsymbol{g}$ is a circular Gaussian with $\sigma=2$ pixels, and the completeness has been estimated on the basis of $10^{4}$ simulated $13 \times 13$ pixels maps assuming a detection threshold corresponding to $\alpha=10^{-3}$.

Although indicative, such experiments, as well those by Ofek \& Zackay (2017), are affected by an important limitation: the comparison between the MF and the PSFF is done assuming 

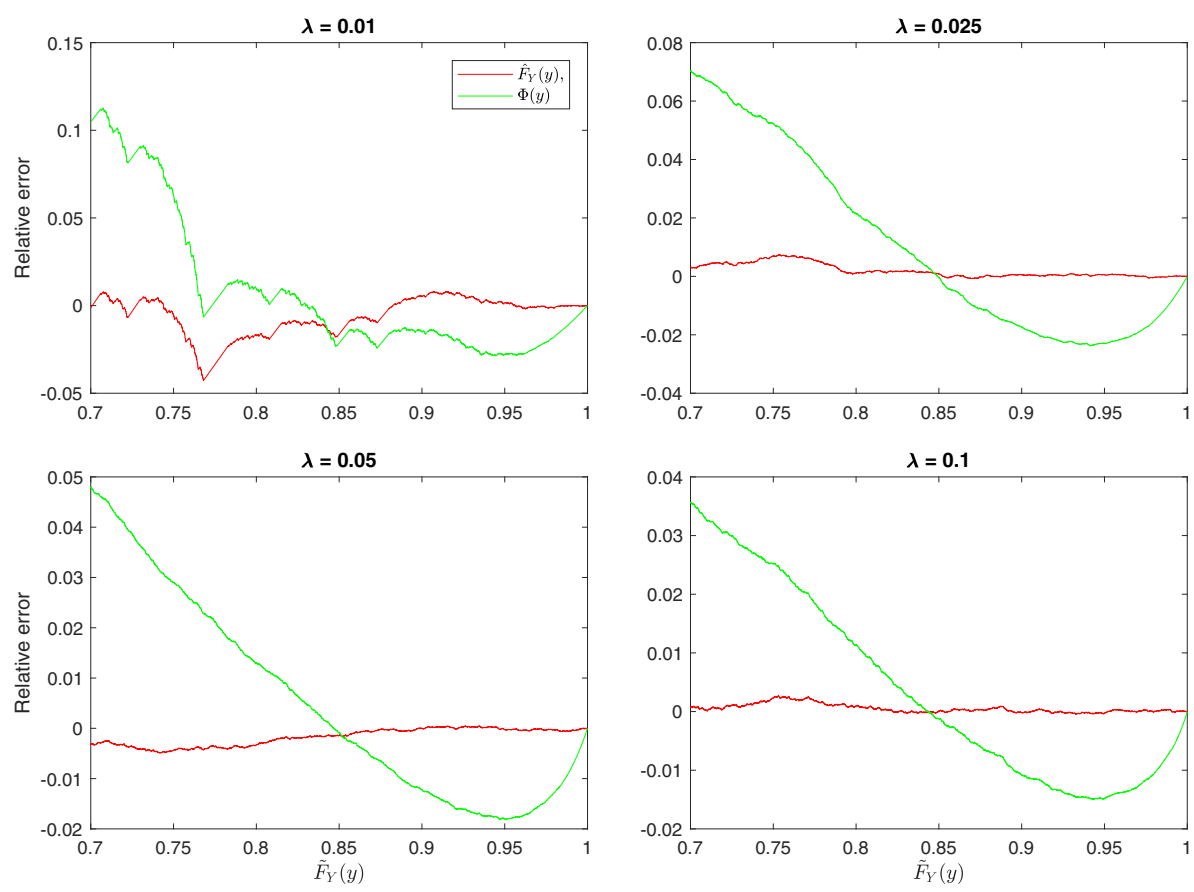

Fig. 3. Relative errors $\left(\hat{F}_{Y}(y)-\tilde{F}_{Y}(y)\right) / \tilde{F}_{Y}(y)$ (red line) and $\left(\Phi(y)-\tilde{F}_{Y}(y)\right) / \tilde{F}_{Y}(y)$ (green line) against $\tilde{F}_{Y}(y)$ for the CDF's in Fig. 2. The very small relative errors of $\hat{F}_{Y}(y)$ highlight its good performance. However, caution has to be used when considering the extreme right end of these diagrams. This is because the sample CDF $\tilde{F}_{Y}(y)$ is not an accurate estimate of $F_{Y}(y)$ when this last is very close to one. Indeed, $F_{Y}(y) \rightarrow 1$ only when $y \rightarrow \infty$ but $\tilde{F}_{Y}\left(y_{\max }\right)=1$ with $y_{\max }$ being the greatest among the simulated random variable $Y$.
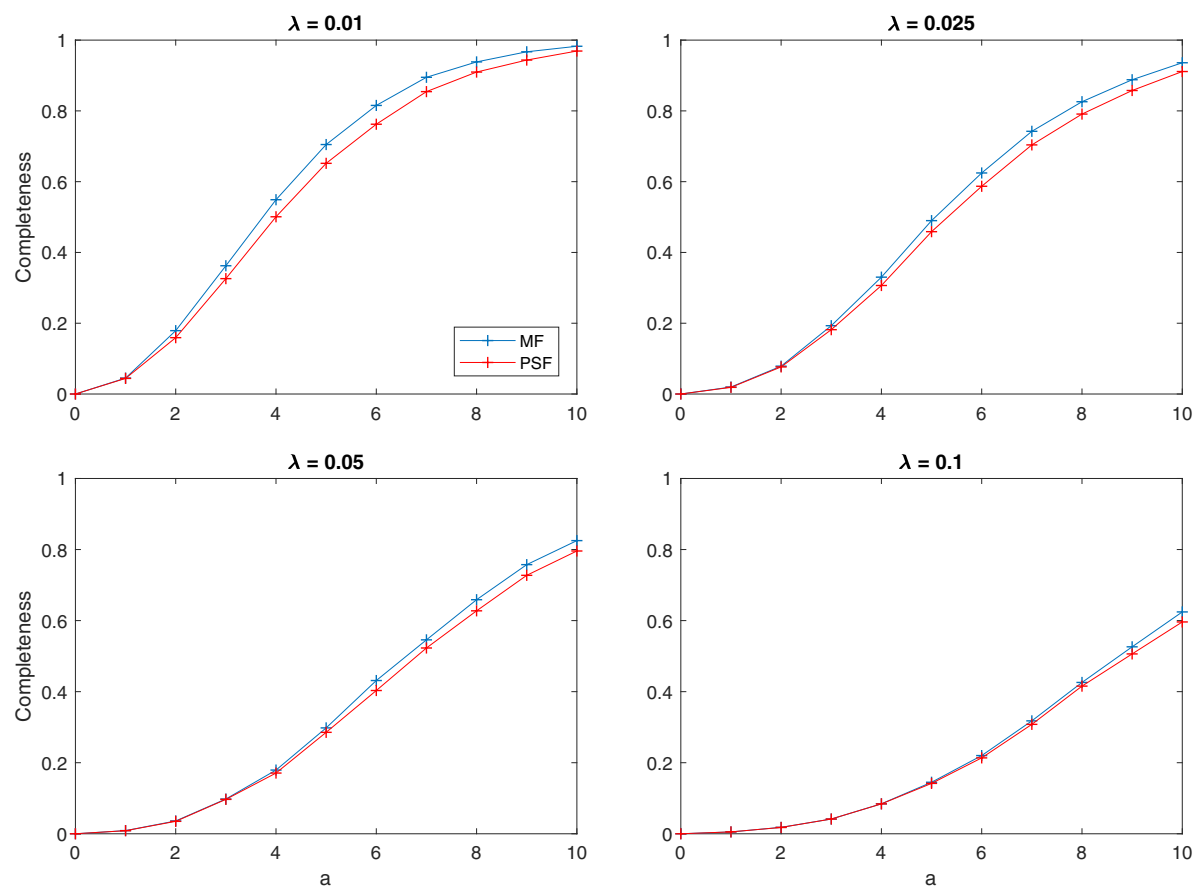

Fig. 4. Results of the numerical experiments presented in Sect. 6. Here, the performances of the MF and of the PSFF are compared on the basis of the completeness (i.e., the fraction of correctly detected signals) for different combinations of the amplitude $a$ of the signal $s$ with the intensity $\lambda$ (units in counts pix $^{-1}$ ) of the background (see Sect. 6). Here, $s$ is given by $a \boldsymbol{g}$ with $\boldsymbol{g}$ a circular Gaussian with $\sigma=2$ pixels normalized to unit volume. $10^{4}$ simulated $13 \times 13$ pixels maps have been used. The detection test has been run assuming a detection threshold corresponding to $\alpha=10^{-3}$.

that $a$ in the MF takes true values. But in real situations this information is not available. An example is sky maps containing point sources with different amplitude. For this reason, we have carried out another set of numerical experiments similar to the previous ones but using incorrect signal amplitudes $a^{*}$. The results are shown in Figs. 5-7 for $a^{*}=0.1,10$, and 100. The indication that comes out from these figures is that, for a given value of $\lambda$, the performances of the MF effectively do not critically depend on the exact value of $a$ and, although less remarkably, tend to remain superior to those provided by the PSFF. Only values of $a^{*}$ that are very different from the true one determine an appreciable degradation of the results.

Finally, another indication which comes out from Figs. 5-7 is that it appears less harmful to use values of $a^{*}$ greater, rather than smaller, the true value. The reason of this fact can be deduced from Fig. 8 which shows the 1-D cut of the MF for different combinations of $\lambda$ and $a$. With $\lambda$ fixed, it is evident that when $a$ increases the filtering action of the MF is stronger. This results in an over-filtered signal but also in a more robust attenuation of the noise. On the contrary, using an $a^{*}$ smaller than $a$ will result in an insufficiently filtered noise. The former situation appears preferable.

\section{Shortcomings of the MF with Poisson noise}

Thanks to the SA it is possible to find a partial solution to a still unsolved problem. In particular, the performances of the MF (Eq. (5)) have been tested under the implicit assumption that the position of $\boldsymbol{s}$ within $\boldsymbol{x}$ is known. In real situations this condition is rarely met (e.g., point sources in a two-dimensional 

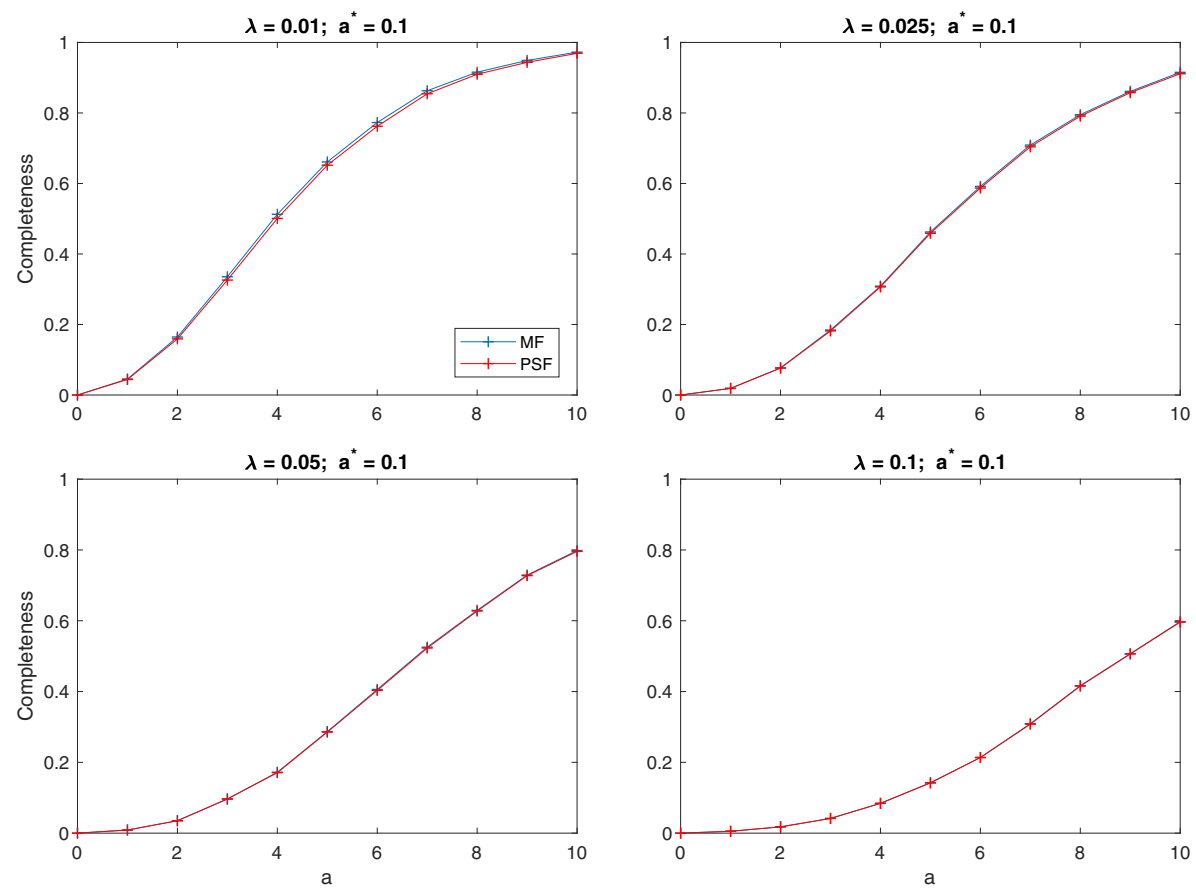

Fig. 5. As in Fig. 4 but the MF has been computed using an incorrect value $a^{*}=0.1$ for the amplitude $a$ of the signal.
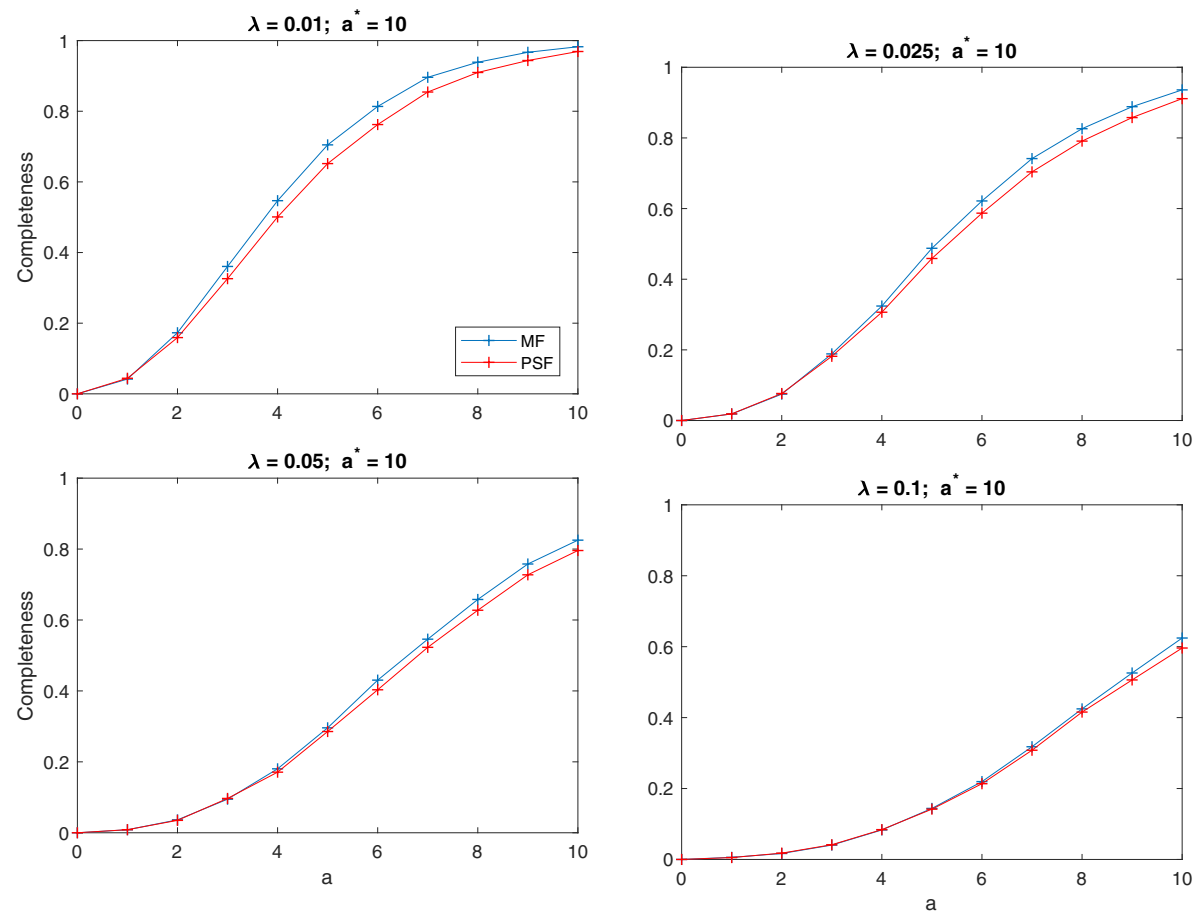

Fig. 6. As in Fig. 4 but the MF has been computed using an incorrect value $a^{*}=10$ for the amplitude $a$ of the signal.

map). The standard procedure to circumvent this issue is to cross-correlate $\boldsymbol{x}$ with the MF and then to apply the detection test Eq. (4) to the resulting most prominent peaks. However, as shown in Vio \& Andreani (2016) and Vio et al. (2017) for the Gaussian case, the PDF of the peaks $\{z\}$ of a random field is different from that of its generic points. Of course, the same holds also for the Poisson case. This is shown by Fig. 9 where the PDF $\hat{f}_{Y}(y)$ of a $2000 \times 2000$ pixels Poisson random field with $\lambda=0.1$ and filtered by means of the MF in Eq. (5) with $s$ a circular Gaussian with $\sigma=2$ pixels, is compared with the histogram $H(z)$ of its peaks. It is evident that working with the peaks, and assuming the PDF $\hat{f}_{Y}(y)$ for the statistic $T(z)$ in Eq. (12), may severely underestimate the PFA with the risk of giving statistical significance to features that belong to the noise.
Contrary to the Gaussian noise (Cheng \& Schwartzman 2015a,b), the PDF of the peaks is not available for the Poisson noise. Without it, a precise computation of the PFA is not possible. Moreover, there is the additional difficulty that, if $N_{p}$ peaks are present in a MF filtered map, then a number $\alpha \times N_{p}$ among them is expected to exceed, by chance, the prefixed detection threshold. For example, if $N_{p}=1000$, then there is a high probability that a detection with a nominal PFA equal to $10^{-3}$ is spurious. Hence, the true PFA has to depend on $N_{p}$ (the lookelsewhere effect). The popular way to get around this issue is to assume $f_{Y}(y)$ as the PDF of the peaks (i.e., the peaks are regarded as generic points of the random field) and then to set PFA $=\alpha / N^{*}$ with $N^{*}$ the number of independent pixels. If the noise is colored, as it happens after the filtering operation, pixels 

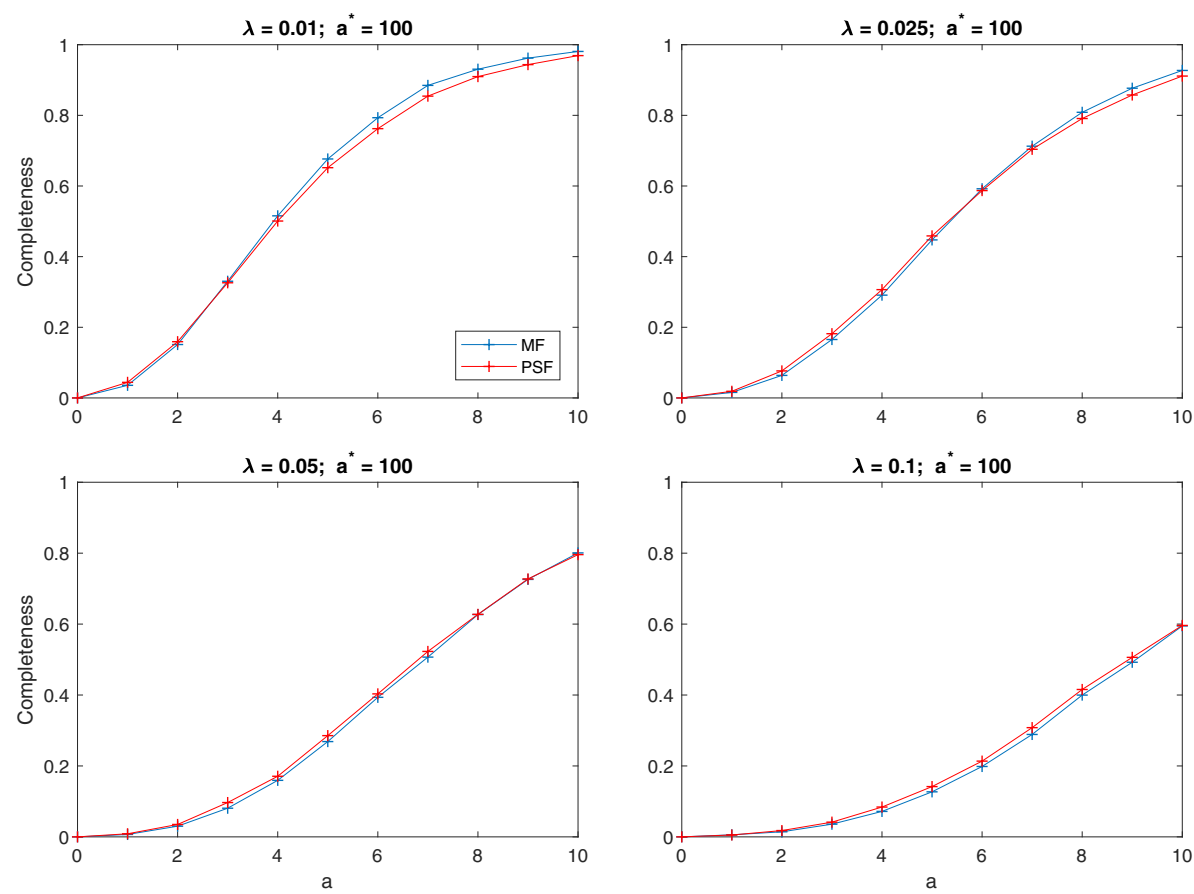

Fig. 7. As in Fig. 4 but the MF has been computed using an incorrect value $a^{*}=100$ for the amplitude $a$ of the signal.
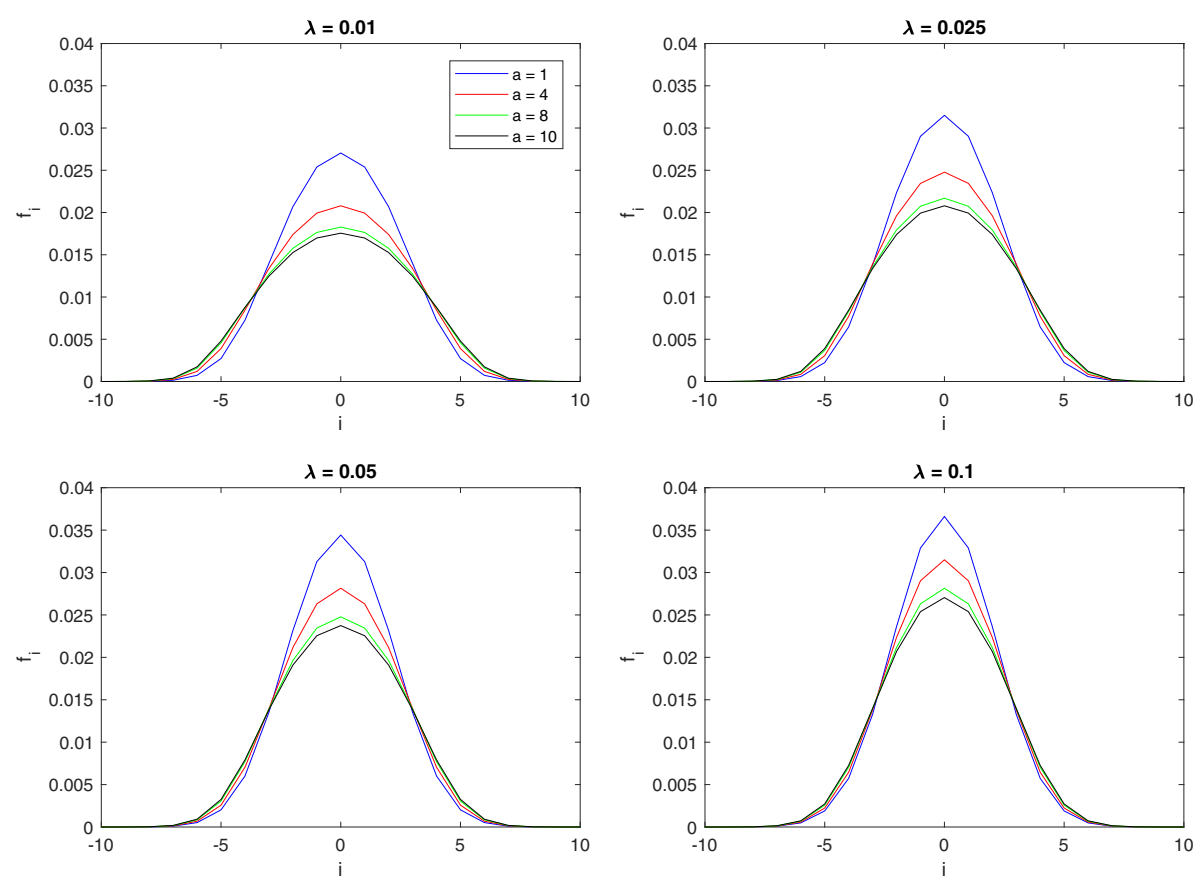

Fig. 8. $1 \mathrm{D}$ cuts of the MF for different values of the signal amplitude $a$ and fixed value of the intensity parameter $\lambda$ of the background.

are correlated with each other. Therefore, $N^{*}$ typically is smaller than $N$ and has to be estimated. Usually the estimation of $N^{*}$ is based on the correlation length of the template $\boldsymbol{g}$. The rational is that pixels with a mutual distance wider than the correlation length can be considered independent. For instance, in the case of a two-dimensional map and $\boldsymbol{g}$ given by a circular Gaussian function with dispersion $\sigma$, Ofek \& Zackay (2017) suggest that $N^{*} \approx N / \sigma^{2}$. This is to point out that such approach provides results which are only correct as an order of magnitude but no alternative is available if additional a priori information is missing.

In the present context, such procedures can be efficiently implemented by means of the order statistics, in particular by exploiting the statistical characteristics of the greatest value of a finite sample of identical and independently distributed (iid) random variables from a given PDF (Hogg et al. 2013). Under the iid condition, the PDF $\psi_{Q}(q)$ and the $\operatorname{CDF} \Psi_{Q}(q)$ of the greatest value $q=y_{\max }$ among a set of $N^{*}$ independent pixels with PDF $\hat{f}_{Y}(y)$ are given by

$\psi_{Q}(q)=N^{*}\left[\hat{F}_{Y}(y)\right]^{N^{*}-1} \hat{f}_{Y}(y)$

and

$\Psi_{Q}(q)=\left[\hat{F}_{Y}(y)\right]^{N^{*}}$,

respectively. Hence, by assuming that the greatest value among a set of pixels coincides with that of a peak, a detection can be claimed when for a given peak it is $1-\Psi_{Q}(q)<\alpha$. 


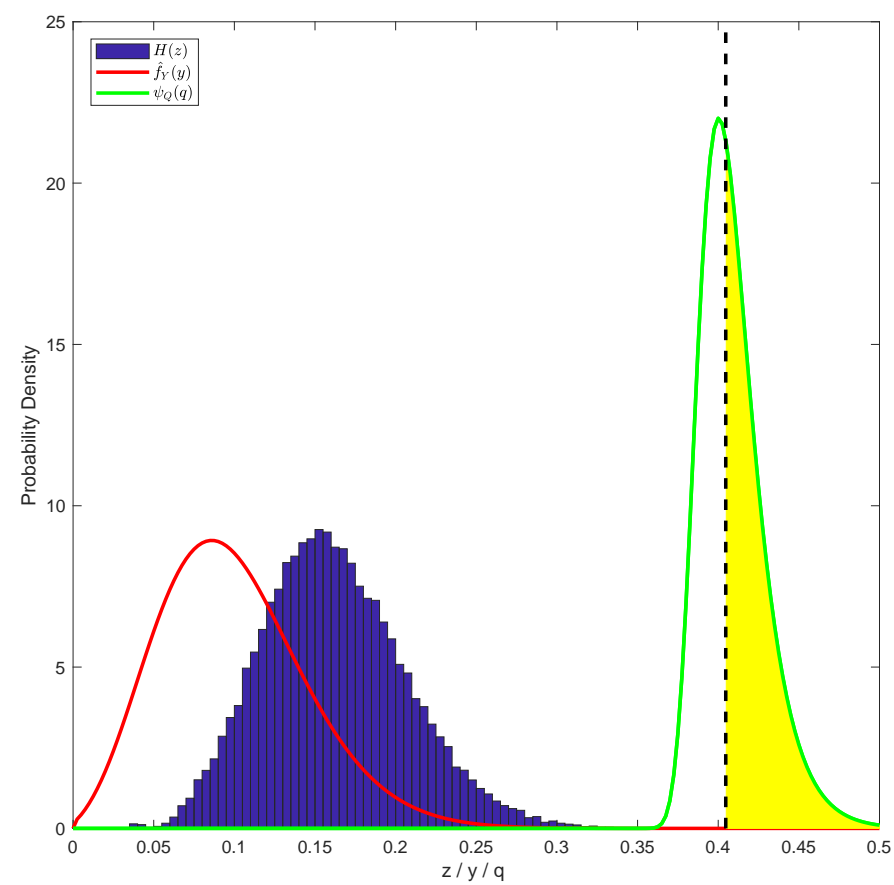

Fig. 9. Comparison of the SA $\hat{f}_{Y}(y)$ (red line) of the PDF of the counts of a MF filtered $2000 \times 2000$ pixels Poisson random field $(\lambda=0.1$ counts pix $^{-1}$ ) with the histogram $H(z)$ of its peaks. The used MF is given by Eq. (5) with $s$ a circular Gaussian with $\sigma=2$ pixels and normalized to unit volume. For reference, the PDF $p_{Z}(z)$ (green line) of the greatest value of the map is also drawn (see text). The color-filled area provides the SPFA for the greatest peak effectively observed in the simulated map $(\mathrm{SPFA} \approx 0.47)$.

This is an alternative way, though equivalent, to threshold the data which does not require the inversion of $\Psi_{Q}(q)$ to fix the parameter $\gamma$ corresponding to a given $\alpha$. In Vio et al. (2017), the quantity $1-\Psi_{Q}(q)$ is called specific probability of false alarm (SPFA). The PDF $\psi_{Q}(q)$ for the numerical experiment of this section is also shown in Fig. 9 as well the SPFA corresponding to the largest peak observed in the map. We stress that in the case of large maps, an alternative based on numerical simulations is not viable.

\section{Conclusions}

In this paper, we have introduced an efficient and effective implementation of the matched filter in the case of low-number count Poisson noise regime. We have shown that although the probability distribution and the cumulative distribution functions of the pixel counts after the MF filtering are not available, they can be approximated with excellent results using the saddlepoint approximation method. With such techniques more accurate estimations of the probability of false detection or false alarm are obtained without making use of empirical or numerical methods.

Acknowledgements. The authors warmly thank Martine Pelzer for her careful reading of the paper and editing of the English.

\section{References}

Briggs, J. 2004, Target Detection by Marine Radar (London: IET)

Butler, R. W. 2007, Saddlepoint Approximations with Applications (Cambridge: Cambridge University Press)

Cheng, D., \& Schwartzman, A. 2015a, Extremes, 18, 213

Cheng, D., \& Schwartzman, A. 2015b, ArXiv e-prints [arXiv: 1503.01328]

Hogg, R. V., McKean, J. W., \& Craig, A. T. 2013, Introduction to Mathematical Statistics (New York: Pearson)

Kay, S. M. 1998, Fundamentals of Statistical Signal Processing: Detection Theory (London: Prentice Hall)

Levy, B. C. 2008, Principles of Signal Detection and Parameter Estimation (New York: Springer Science+Business Media)

Lugannani, R., \& Rice, S. O. 1980, Adv. Appl. Proban., 12, 475

Macmillan, N. A., \& Creelman, C. D. 2005, Detection Theory: A User's Guide (Mahwah: Lawrence Erlbaum Associates)

Minkof, J. 2002, Signal Processing Fundamentals and Applications for Communications and Sensing (Boston: Artech House)

Ofek, E. O., \& Zakay, B. 2018, AJ, 155, 1690

Osche, g. R. 2002, Optical Detection Theory for Laser Applications (Hoboken: Wiley-Interscience)

Paolella, M. S. 2007, Intermediate Probability (Chichester: John Wiley \& Sons Ltd)

Stewart, I. M. 2006, A\&A, 454, 1009

Tuzlukov, V. P. 2001, Signal Detection Theory (New York: Springer Science+ Business Media)

Vio, R., \& Andreani, P. 2016, A\&A, 589, A20

Vio, R., Vergès, C., \& Andreani, P. 2017, A\&A, 604, A115

Yao, K., Lorenzelli, F., \& Chen, C. E. 2013, Detection and Estimation for Communication and Radar Systems (Cambridge: Cambridge University Press) 


\section{Appendix A: Simple derivation of the saddlepoint approximation}

Saddlepoint approximation (SA) is a powerful tool for obtaining accurate expressions of probability density functions and cumulative distribution functions. It can be derived in a number of ways. Most of them, however, are rather technical. Here, we provide an informal derivation due to Paolella (2007). A more technical and rigorous derivation can be found in Butler (2007).

As seen in Appendix B, if $X$ is a random variable with PDF $f_{X}(x)$, moment generating function (MGF) $M_{X}(s)$ existing for $s \in U$ with $U$ a neighborhood around zero, and cumulant generating function (CGF) $K_{X}(s)=\ln M_{X}(s)$, its mean and variance are given by $K_{X}^{(1)}(0)$ and $K_{X}^{(2)}(0)$, respectively. With this premise, the derivation of the SA proceeds according to the following steps:

- a new random variable $T_{s}$ is defined having PDF

$$
f_{T_{s}}(x ; s)=\frac{\mathrm{e}^{x s} f_{X}(x)}{M_{X}(s)},
$$

for some $s \in U$;

- the MGF and the CGF of $T_{s}$ are computed, respectively, as

$$
\begin{aligned}
M_{T_{s}}(t) & =\frac{M_{X}(t+s)}{M_{X}(s)}, \\
K_{T_{s}}(t) & =K_{X}(t+s)-K_{X}(s) .
\end{aligned}
$$

The mean and the variance of $T_{s}$ are given, respectively, by $K_{T}^{(1)}(0)=K_{X}^{(1)}(s)$ and $K_{T}^{(2)}(0)=K_{X}^{(2)}(s)$;

- fixed an arbitrary $s_{0} \in U, f_{T_{s}}(x ; s)$ is approximated by a Gaussian PDF $\phi\left(x ; \mu_{0}, \sigma_{0}^{2}\right)$ with mean $\mu_{0}$ and variance $\sigma_{0}^{2}$. Here, $\mu_{0}=K_{X}^{(1)}\left(s_{0}\right)$ and $\sigma_{0}^{2}=K_{X}^{(2)}\left(s_{0}\right)$;

- from Eq. (A.1) $f_{X}(x)$, can be approximated by means of the $\operatorname{PDF} \hat{f}_{X}(x)$

$$
\begin{aligned}
\hat{f}_{X}(x) & =\phi\left(x ; \mu_{0}, \sigma_{0}^{2}\right) M_{X}\left(s_{0}\right) \mathrm{e}^{-s_{0} x}, \\
& =\frac{1}{\sqrt{2 \pi \sigma_{0}^{2}}} \exp \left\{-\frac{1}{2 \sigma_{0}^{2}}\left(x-\mu_{0}\right)^{2}\right\} M_{X}\left(s_{0}\right) \mathrm{e}^{-s_{0} x} ;
\end{aligned}
$$

- the value of $s_{0}$ has to be determined in such a way that, for a fixed $x, \hat{f}_{X}(x)$ is the best approximation to $f_{X}(x)$. In this respect, it is well known that the normal approximation to the distribution of a random variable $X$ is accurate near the mean of $X$, but degrades in the tails. As a consequence, it is logical to choose a value $\hat{s}$ of $s_{0}$ such as $\mu_{0}=x$, i.e.,

$K_{X}^{(1)}(\hat{s})=x ;$

- once $\hat{s}$ has been fixed, the normal density approximation $\phi\left(x ; \mu_{0}, \sigma_{0}^{2}\right)$ becomes $\phi\left(x ; K_{X}^{(1)}(\hat{s}), K_{X}^{(2)}(\hat{s})\right)$ and the approximation to $f_{X}(x)$ simplifies to

$\hat{f}_{X}(x)=\frac{1}{\sqrt{2 \pi K_{X}^{(2)}(\hat{s})}} \exp \left\{K_{X}(\hat{s})-x \hat{s}\right\}, \quad x=K_{X}^{(1)}(\hat{s})$.
The only critical point in this procedure is that $\hat{s}$ has to be evaluated, via the solution of Eq. (A.6), for each $x$ for which $\hat{f}_{X}(x)$ has to be computed. In general, this requires a numerical approach. However, for a fixed $x$ Eq. (A.6) has only one solution $\hat{s}$ since it can be shown that $K_{X}(s)$ is always a strictly convex function when evaluated over the converge interval $U$ of $M_{X}(s)$. Consequently, the mapping $K^{(1)}(s)$ for $s \in U$ into the support of the PDF $f_{X}(x)$ is a bijection and thereof $K^{(1)}(s)$ is strictly increasing (see p. 6 in Butler 2007). Another question is that $\hat{f}_{X}(x)$ will not, in general, integrate to one, although it will usually not be far off. Therefore, it has to be numerically normalized.

\section{Appendix B: The moment and cumulant generating functions}

Given a continuous random variable $X$ with probability density function $f_{X}(x)$ and a positive number $h$ such that for $-h<s<h$ the mathematical expectation

$E\left[\mathrm{e}^{s X}\right]=\int_{-\infty}^{+\infty} \mathrm{e}^{s x} f_{X}(x) \mathrm{d} x$

exists, then this expectation is called the moment generating function (MGF) of $X$ and is denoted by $M_{X}(s)$. The largest open interval $U=(a, b)$ around zero where $M_{X}(s)<\infty$ for $s \in U$ is referred as the convergence strip of $M_{X}(s)$. In the case $X$ is a discrete random variable with discrete $\operatorname{PDF} F_{X}(k), M_{X}(s)$ becomes

$M_{X}(s)=\sum_{k=-\infty}^{\infty} \mathrm{e}^{s k} f_{X}(k), \quad s \in U$.

The cumulant generating function (CGF) of $M_{X}(x)$ is defined as

$K_{X}(s)=\ln M_{X}(s), \quad s \in U$.

The terms $\kappa_{i}$ in the series expansion $K_{X}(t)=\sum_{r=0}^{\infty} k_{r} t^{r} / r$ ! are referred to as the cumulants of $X$ so that the $i$ th derivative of $K_{X}(t)$ evaluated at $t=0$ is $\kappa_{i}$, i.e.,

$\kappa_{i}=\left.K_{X}^{(i)}(t)\right|_{t=0}$.

It is straightforward to show that $\kappa_{1}=\mu=E[X]$ and $\kappa_{2}=\sigma^{2}=$ $E\left[X^{2}-\mu^{2}\right]$, meaning that the first two cumulants provide the mean and the variance of $X$.

One important property of the CGF is that in the case of a sequence of independent random variable $X_{1}, X_{2}, \ldots, X_{N}$ for which $X_{i}$ has CGF $K_{i}$ determined over $U_{i}=\left(a_{i}, b_{i}\right)$, then the CGF of $X=\sum_{i=1}^{N} X_{i}$ is

$K_{X}(s)=\sum_{i=1}^{N} K_{X_{i}}(s), \quad s \in\left(\max a_{i}, \min b_{i}\right)$.

In other words, the CGF of a sum of independent random variable is given by the sum of the respective CGFs. 